\title{
Plasma Level and Expression of Visfatin in the Porcine Hypothalamus During the Estrous Cycle and Early Pregnancy
}

\section{Tadeusz Kaminski ( $\nabla$ tkam@uwm.edu.pl)}

University of Warmia and Mazury in Olsztyn: Uniwersytet Warminsko-Mazurski

Marta Kiezun

University of Warmia and Mazury

Ewa Zaobidna

University of Warmia and Mazury

Kamil Dobrzyń

University of Warmia and Mazury

\section{Barbara Wasilewska}

University of Warmia and Mazury in Olsztyn: Uniwersytet Warminsko-Mazurski

\section{Ewa Mlyczynska}

Jagiellonian University: Uniwersytet Jagiellonski w Krakowie

\section{Edyta Rytelewska}

University of Warmia and Mazury

Katarzyna Kisielewska

University of Warmia and Mazury

Marlena Gudelska

University of Warmia and Mazury

Kinga Bors

University of Warmia and Mazury

Grzegorz Kopij

University of Warmia and Mazury

Karolina Szymanska

University of Warmia and Mazury

\section{Barbara Kaminska}

University of Warmia and Mazury

\section{Agnieszka Rak}

Jagiellonian University

\section{Nina Smolinska}

University of Warmia and Mazury 
Research

Keywords: visfatin, hypothalamus, estrous cycle, early pregnancy, pig

Posted Date: October 20th, 2020

DOI: https://doi.org/10.21203/rs.3.rs-93399/v1

License: (c) (i) This work is licensed under a Creative Commons Attribution 4.0 International License. Read Full License 
3

4 5

Plasma level and expression of visfatin in the porcine hypothalamus during the estrous cycle and early pregnancy

Tadeusz Kaminski ${ }^{1 *}$, Marta Kiezun ${ }^{1}$, Ewa Zaobidna ${ }^{1}$, Kamil Dobrzyn $^{1}$, Barbara Wasilewska ${ }^{3}$, Ewa Młyczyńska ${ }^{2}$, Edyta Rytelewska ${ }^{1}$, Katarzyna Kisielewska ${ }^{1}$, Marlena Gudelska ${ }^{1}$, Kinga

Bors ${ }^{1}$, Grzegorz Kopij ${ }^{1}$, Karolina Szymańska ${ }^{1}$, Barbara Kaminska ${ }^{1}$, Agnieszka Rak ${ }^{2}$ and Nina Smolińska ${ }^{1}$

${ }^{1}$ Department of Animal Anatomy and Physiology, Faculty of Biology and Biotechnology, University of Warmia and Mazury in Olsztyn, Oczapowskiego St. 1A, 10-719 Olsztyn-Kortowo, Poland

${ }^{2}$ Department of Physiology and Toxicology of Reproduction, Institute of Zoology and Biomedical Research, Jagiellonian University in Krakow, Gronostajowa St. 9, 31-387 Krakow, Poland.

${ }^{3}$ Department of Human Physiology and Pathophysiology, School of Medicine, University of Warmia and Mazury in Olsztyn, Warszawska St. 30, 10-082 Olsztyn

Tadeusz Kaminski (TK), tkam@uwm.edu.pl

Marta Kiezun (MK), marta.kiezun@uwm.edu.pl

Ewa Zaobidna (EZ), ewa.zaobidna@uwm.edu.pl

Kamil Dobrzyn (KD), kamil.dobrzyn@uwm.edu.pl

Barbara Wasilewska (BW), barbara.wasilewska@uwm.edu.pl

Ewa Mlyczynska(EM), ewa.mlyczynska@alumni.uj.edu.pl

Edyta Rytelewska(ER), edyta.rytelewska@uwm.edu.pl

Katarzyna Kisielewska(KK), katarzyna.kisielewska@uwm.edu.pl

Marlena Gudelska(MG), marlena.gudelska@uwm.edu.pl

Kinga Bors (KB), kinga.bors@uwm.edu.pl

Grzegorz Kopij (GK), grzegorz.kopij@uwm.edu.pl

Karolina Szymańska (KS), karolina.szymanska@uwm.edu.pl

Barbara Kaminska (BK), barbara.kaminska@uwm.edu.pl

Agnieszka Rak (AR), agnieszka.rak@uj.edu.pl

Nina Smolinska (NS), nina.smolinska@uwm.edu.pl

* Corresponding author: Tadeusz Kaminski, tkam@uwm.edu.pl, tel. +48 895233201 , Department of Animal Anatomy and Physiology, Faculty of Biology and Biotechnology, 
36 University of Warmia and Mazury in Olsztyn, Oczapowskiego St. 1A, 10-719 Olsztyn-

37 Kortowo, Poland

38

39

40

Ethics approval and consent to participate

41 Not applicable.

42

Consent for publication

44 Not applicable.

45

Availability of data and materials

47 The datasets analysed during the current study are available from the corresponding author on

48 reasonable request.

49

\section{Competing interests}

51 The authors declare that they have no competing interests.

52

53

Funding

54 This research was supported by the National Science Centre (Project no.: 55 2018/31/B/NZ9/00781).

57 Authors' contributions

58 TK, NS and AR contributed to the conception and design. TK contributed to article writing. $59 \mathrm{NS}, \mathrm{AR}$ and EZ contributed to revising the article. MK, KD, BW, EZ, EM, ER, KK, MG, KB, $60 \mathrm{GK}, \mathrm{KS}$ and BK contributed to data collection. The authors read and approved the final 61 version of the manuscript.

62

Acknowledgements

64 Not applicable.

65

Authors' information (optional)

67 Not applicable. 


\section{Abstract}

Background: Visfatin exists in two forms: the intracellular form which is a rate limiting enzyme engaged in nicotinamide adenine dinucleotide biosynthesis and the extracellular form considered as an adipokine, produced mainly by the adipose tissue. Visfatin could be an energy sensor involved in regulating female fertility, which creates a hormonal link integrating the control of energy homeostasis and reproduction.

Methods: The study compares the expression levels of visfatin gene (quantitative real time PCR) and protein (Western blotting and fluorescent immunohistochemistry) in the selected areas of the porcine hypothalamus responsible for gonadotropin releasing hormone synthesis: the mediobasal hypothalamus $(\mathrm{MBH})$ and preoptic area (POA), and visfatin concentrations in the blood plasma (enzyme-linked immunosorbent assay). The tissue samples were harvested from gilts on days 2-3, 10-12, 14-16 and 17-19 of the estrous cycle, and on days 10-11, 12-13, 15-16, 27-28 of pregnancy. Differences between groups were analyzed by one-way ANOVA followed by Tukey's post hoc test.

Results: During the estrous cycle, visfatin mRNA expression in the MBH was higher on days 2-3 and 17-19, while the visfatin protein concentration on days 17-19. During early pregnancy, the most pronounced gene and protein expression in the MBH was found on days 15-16 and 10-11, respectively. In the POA during the estrous cycle, visfatin gene expression was the highest on days 17-19, and the protein level of visfatin on days 14-16. During early pregnancy, the highest expression of visfatin gene in the POA was observed on days 15-16, whereas the protein concentrations on days 27-28. Blood plasma concentrations of visfatin during the estrous cycle were higher on days 2-3 in relation to other studied phases of the cycle. During early pregnancy, the highest visfatin contents in the blood plasma were observed on days 12-13. Visfatin gene and protein expression in MBH and POA, and visfatin plasma concentrations differed during early pregnancy in relation to days 10-12 of the cycle.

Conclusions: This study demonstrated visfatin expression in the porcine hypothalamus and its dependence on hormonal milieu related to the estrous cycle and early pregnancy.

Keywords: visfatin, hypothalamus, estrous cycle, early pregnancy, pig 


\section{Background}

Visfatin, also termed nicotinamide phosphoribosyltransferase (NAMPT), was identified in 2005 by Fukuhara et al. [1]. It exists in two forms: the intracellular form which is a rate limiting enzyme engaged in nicotinamide adenine dinucleotide (NAD) biosynthesis from nicotinamide and the extracellular form considered as an adipokine. Until now no visfatin receptor has been identified. On the other hand, it is suggested that visfatin can bind and activate insulin receptor (INSR), and downstream signaling pathways [2]. The adipokine and insulin do not compete for binding to INSR which implies that these two hormones recognize different regions of the receptor [3]. Apart from the adipose tissue, visfatin was also expressed in tissues creating the hypothalamic-pituitary-gonadal (HPG) axis: in the mouse hypothalamus [4], in the pituitaries collected from sheep [5] and mice [4], in the ovarian follicular cells of humans, cows and mice [4, 6-8] as well as bovine corpora lutea [8].Visfatin was also found in the porcine tissues, including the pituitary and ovary [9]. Plasma and perirenal adipose tissue NAMPT levels were not different between the two breeds of pigs fat Meishan and lean Large White, suggesting that this adipokine is not involved in the fattening in swine [10]. The occurrence of visfatin mRNA and protein in various tissues suggests that non-adipose tissue may also contribute to serum visfatin levels. Visfatin expression in the adipocytes can be affected by hormonal factors such as the steroid hormones, tumor necrosis factor $\alpha(\mathrm{TNF} \alpha)$, growth hormone (GH) and dexamethasone $[9,11,12]$, while in the human granulosa cells by human chorionic gonadotropin (hCG) and prostaglandin $\mathrm{E}_{2}$ [6]. It is suggested that visfatin gene expression can be controlled by species-specific regulatory mechanism [9] and the adipokine concentrations in human adipose tissue are affected by hormonal status related to pregnancy [13]. The circumstances showing that hormonal milieu typical for pregnancy may affect visfatin production are demonstrated in a study by Mastorakos et al. [14] indicating the increase in visfatin plasma concentrations with advancing gestational age of women. However, the effect of hormonal changes associated with the individual phases of the estrous cycle and periods of pregnancy on the levels of visfatin transcript and protein has not been analyzed in the hypothalamus.

Visfatin is likely to have pleiotropic properties. It plays a role in the control of energy homeostasis, inflammation and cell differentiation. Circulating visfatin concentrations correlate positively with body mass index and obesity $[15,16]$. As an insulin-mimetic hormone visfatin stimulates glucose uptake in muscle cells and adipocytes [17] and suppresses glucose release from hepatocytes [18]. Visfatin, like other adipokines [19-22], could be an energy sensor involved in regulating female fertility. Administration of visfatin 
during the superovulation of mice increased developmental competence of oocytes and

142 fertility potential [23]. In women undergoing ovarian stimulation, a correlation was found between visfatin concentration in the ovarian follicular fluids and the number of oocytes retrieved [6]. An effect of visfatin on the ovarian steroidogenesis was also demonstrated $[7,8]$. It also seems that visfatin plays an important role in the implantation and placentation [17, 24]. It is plausible that the action of visfatin during the estrous cycle and early pregnancy (maternal recognition of pregnancy, implantation, placentation) is partly achieved through its influence on the endocrine HPG axis, including the hypothalamus, what has not been investigated so far.

Visfatin may act through the regulation of the hypothalamic structures secreting gonadotropin releasing hormone $(\mathrm{GnRH})$ - the key factor controlling the pituitary and ovaries. We hypothesized that hypothalamic visfatin expression is dependent on animal hormonal status. Therefore, the goal of the present study was to investigate visfatin gene and protein expression in the porcine hypothalamic structures: mediobasal hypothalamus (MBH) and preoptic area (POA) engaged in GnRH generation, and plasma visfatin concentrations during the estrous cycle and early pregnancy.

\section{Methods}

\subsection{Animals and tissues collection}

Tissue samples were harvested from animals intended for commercial slaughter and meat processing. The animals used in the study were maintained and transported under conditions defined in the Act of $15^{\text {th }}$ January 2015 (Poland) on the protection of animals used for scientific or educational purposes. Mature cross-breed gilts (Large White x Polish Landrace) aged 7 to 8 months and weighing 140 to $150 \mathrm{~kg}$, obtained from the private breeding farm, were used in the study. Diet was balanced (crude protein, metabolizable energy, exogenous amino acids and minerals) in accordance with the Polish nutritional standards for domestic pigs, access to freshwater ad libitum. To investigate the visfatin expression, a total of forty animals were divided into eight experimental groups ( $\mathrm{n}=5$ per group), as follows: days $2-3$ (early-luteal phase, the presence of corpora haemorrhagica), 10-12 (mid-luteal phase, the phase when the corpus luteum activity is the highest and similar to that noted during pregnancy), 14-16 (late-luteal phase, the phase of luteolysis) and 17-19 (follicular phase) of the estrous cycle, as well as days 10-11 (migration of the embryos within the uterus), 12-13 (maternal recognition of pregnancy), 15-16 (beginning of implantation) and 27-28 (end of implantation) of pregnancy. Gilts were monitored daily for an estrus behavior in the presence 
of a boar. The day of the onset of the second estrus was marked as day 0 of the estrous cycle. The phase of the estrous cycle was also confirmed based on the morphology of ovaries [25]. In the case of pregnant pigs, natural insemination was performed on days 1-2 of the estrous cycle. In the case of days 15-16 and 27-28 of pregnancy, the stage of pregnancy was also confirmed by the presence and morphology of conceptuses/trophoblasts [26]. Within a few minutes after slaughter, blood samples were collected into heparinized tubes and centrifuged at $2500 \times \mathrm{g}$ for $15 \mathrm{~min}$ at $4^{\circ} \mathrm{C}$. The obtained plasma was stored at $-80^{\circ} \mathrm{C}$ for further measurement. Subsequently, the hypothalamus was removed and the MBH and POA were excised as previously described [27]. The mediobasal hypothalamus was defined as a block of tissue bounded rostrally by the optic chiasma, caudally by the mammillary body, laterally by the hypothalamic sulci and dorsally by a $5 \mathrm{~mm}$-deep cut. The preoptic area was limited rostrally approximately $5 \mathrm{~mm}$ anterior to the optic chiasma and caudally by the rostral border of the MBH. The mediobasal hypothalamus and POA obtained on days 15-16 of pregnancy and 10-12 of the cycle were divided into two parts, from which one half was intended for immunofluorescent staining (placed in $4 \%$ buffered paraformaldehyde; $\mathrm{pH}=7.4,4^{\circ} \mathrm{C}$ ), and the other half was frozen in liquid nitrogen and stored at $-80^{\circ} \mathrm{C}$ until processing for RNA and protein analyzis.

\subsection{The analyzis of visfatin localization in the porcine hypothalamus using fluorescent} immunohistochemistry

The tissue blocks were fixed by immersion for $36 \mathrm{~h}$ in $4 \%$ buffered paraformaldehyde $\left(\mathrm{pH}=7.4 ; 4^{\circ} \mathrm{C}\right)$. Following fixation, the brains were washed in $0.1 \mathrm{M}$ phosphate-buffered saline (PBS) and then cryoprotected for 3-6 days in graded solutions (19\% and 30\%) of sucrose (Sigma-Aldrich, USA) at $4{ }^{\circ} \mathrm{C}$ until fully infiltrated. The tissues were frozen and cut into $12 \mu \mathrm{m}$ thick cryostat coronal sections and stored at $-80^{\circ} \mathrm{C}$. Localization of the hypothalamic nuclei was calculated based on Felix et al. [28]. Frozen brain sections were processed for routine single-immunofluorescence labelling. All steps of the staining procedures were conducted in humid chambers at room temperature. The sections were airdried for $30 \mathrm{~min}$, washed 3 times in PBS and incubated for $1 \mathrm{~h}$ with blocking buffer $(0.1 \mathrm{M}$ PBS, 10\% normal horse serum, 0.01\% BSA (bovine serum albumin), 1\% Tween, 0.05\% thimerosal, $0.01 \% \mathrm{NaN}_{3}$ ). Then, the sections were incubated overnight with rabbit polyclonal antibodies against visfatin (1:600; cat.-no. ab233294, Abcam, UK). Following subsequent rinsing in PBS $(3 \times 15 \mathrm{~min})$, the sections were incubated $(1 \mathrm{~h})$ with the Alexa Fluor 555 donkey anti-rabbit antibodies (1:1000; cat.-no. A-31572, Thermo Fisher Scientific, USA). After that, slides were washed in PBS and coverslipped in buffered carboxyglycerol 
211 ( $\mathrm{pH}=8.6)$. The sections were analyzed with an Olympus BX51 microscope equipped with an

212 Olympus XM10 digital camera (Tokyo, Japan). Images were acquired with cellSens

213 Dimension 1.7 Image Processing software (Olympus Soft Imaging Solutions, Münster,

214 Germany). Standard controls, i.e. the omission and replacement of primary antisera by non215 immunosera were applied to test antibody and method specificity. Lack of any 216 immunoreactions indicated specificity.

\subsection{The analyzis of NAMPT gene expression in the porcine hypothalamus using} quantitative real-time PCR ( $q P C R)$

Total RNA was isolated using the TRI reagent (Sigma-Aldrich, USA) according to the manufacturer's instructions. The quantity and quality of the isolated RNA were determined spectrophotometrically (Infinite M200 Pro, Tecan, Switzerland). One microgram of RNA was reverse transcribed into cDNA using the Omniscript RT Kit (Qiagen, Germany) with $0.5 \mu \mathrm{g}$ oligo(dt) $)_{15}$ primer (Roche, Germany) in a total volume of $20 \mu \mathrm{l}$ at $37^{\circ} \mathrm{C}$ for $1 \mathrm{~h}$ and then terminated at $93^{\circ} \mathrm{C}$ for $5 \mathrm{~min}$. Quantitative real-time PCR (qPCR) analyzis was performed using an AriaMx Real-Time PCR System (Agilent Technologies, USA) with Power SYBR Green Master Mix (Applied Biosystems Inc., USA), as described previously [29]. Specific primer pairs used to amplify parts of $N A M P T, U B C$ (ubiquitin C) and $18 s R N A$ (18S ribosomal RNA) genes are detailed in the Table 1 . The $U B C$ and $18 s R N A$ were used as reference genes. Preliminary studies confirmed that their expressions were stable in both studied tissues during all the studied stages of the estrous cycle and early pregnancy (similar $\mathrm{Ct}$ values without statistically significant differences). The qPCR reaction mixtures at the final volume of $20 \mu 1$ contained $20 \mathrm{ng}$ of cDNA, the appropriate forward and reverse primers at the concentrations detailed in the Table 1, $12.5 \mu$ l of Power SYBR Green PCR Master Mix (Applied Biosystems, USA) and RNase free water. The qPCR conditions are detailed in the Table 1. Negative controls contained RNase free water instead of cDNA. All reactions were amplified in duplicates. The analyzis of melting curve was used to confirm the specificity of amplification. The relative gene expression level was calculated using the comparative cycle threshold method $(\Delta \Delta \mathrm{Ct},[30])$ and normalized using the geometrical means of reference genes expression levels of $U B C$ and $18 s R N A$. The $\mathrm{Ct}$ values for all non-template controls were under the detection threshold. 
About 30-mg-pieces of porcine MBHs and POAs were homogenized in $500 \mu 1$ of T-PER Tissue Protein Extraction Reagent (Thermo Fisher Scientific, USA). Equal amounts of lysate (50 $\mu \mathrm{g}$ protein/sample) were separated in Mini-Protean TGX System Precast Protein Gels (Bio-Rad Laboratories Inc., USA) and then transferred to Trans-Blot Turbo Mini PVDF Transfer Packs (Bio-Rad Laboratories Inc., USA). The membranes were blocked for $1 \mathrm{~h}$ in $0.02 \mathrm{M}$ Tris-buffered saline containing 5\% BSA and $0.1 \%$ Tween 20, then incubated overnight at $4^{\circ} \mathrm{C}$ with primary anti-visfatin antibody (cat.-no. ab233294; Abcam, UK) diluted at 1:700. Subsequently, the membranes were washed with TBST (Tris-buffered saline containing $0.1 \%$ Tween ${ }^{\circledR} 20$ Detergent) and incubated for $1 \mathrm{~h}$ at room temperature with a horseradish peroxidase-conjugated antibody (cat.-no. 7074; Cell Signaling Technology, USA) diluted at 1:1000. The anti- $\beta$-actin antibodies (cat.-no. A5316; Sigma-Aldrich, USA) diluted at 1:5000 were used as a loading control. Signals were detected by chemiluminescence using the Western blotting Luminol Reagent (Advansta Inc., USA), and visualized using the Chemidoc $^{\mathrm{TM}}$ XRS + System (BioRad Laboratories Inc., USA). All visible bands were quantified using a densitometer and ImageJ software (US National Institutes of Health, USA).

2.5. The analyzis of visfatin concentration in the porcine blood plasma using enzyme-linked immunosorbent assay (ELISA)

The concentrations of visfatin protein in plasma were determined using a commercial ELISA kit (cat.-no. MBS736963, MyBioSource, USA) according to the manufacturer's protocol. The range of standard curve was 0 to $50 \mathrm{ng} / \mathrm{mL}$. The sensitivity of the assay, defined as the least protein concentration that could be differentiated from zero samples, was $<0.1$ $\mathrm{ng} / \mathrm{mL}$. According to the manufacturer, no significant cross-reactivity or interference between visfatin and homologous proteins assayed has been observed. Absorbance values were measured at $450 \mathrm{~nm}$ using an Infinite M200 Pro reader with Tecan i-control software (Tecan, Switzerland). The data were linearised by plotting the log of visfatin concentrations versus the $\log$ of the optical density and the best fit line was determined by regression analyzis. Intraassay coefficient of variation of the ELISA assay for visfatin was $5.29 \%$.

\subsection{Data analyzis}

All experimental data were presented as means \pm standard error of the mean (SEM) from five different observations. Differences between groups were analysed by one-way ANOVA followed by Tukey's honest significant difference post hoc test. Statistical analyses were performed using Statistica Software (StatSoft Inc., Tulsa, USA). Values for $p<0.05$ were considered as statistically significant. 


\section{Results}

282

\subsection{The distribution of visfatin in the porcine hypothalamus}

The immunofluorescence staining has shown the presence of visfatin in some regions of the pig hypothalamus both during the estrous cycle (days 10-12 - the mid-luteal phase; Fig. 1) as well as during early gestation (days 15-16 of pregnancy - the beginning of implantation; Fig. 2). In the supraoptic nucleus (SON), periventricular nucleus (PPN), sexually dimorphic nucleus of the preoptic area $(\mathrm{SDN})$, paraventricular nucleus $(\mathrm{PVN})$, and additionally the diagonal band of Broca (DBB), a part of basal forebrain, visfatin showed very intensive immunoreactivity and it was usually confined to the the nucleus as well as neuroplasm of the cell bodies. Weaker immunoreactivity was observed in the lateral (LPA) and medial preoptic area (MPA). In the suprachiasmatic nucleus (SCN), ventromedial nucleus (VMH) and arcuate nucleus (ARC) visfatin was located in the nucleus, sporadically was found in cell neuroplasm.

\subsection{Gene and protein expression of visfatin in the mediobasal hypothalamus}

During the estrous cycle, visfatin gene expression was higher on days 2-3 and 17-19 in comparison to days 14-16. In turn, the protein concentration of visfatin was the highest on days 17-19 compared to days 2 to 12 ( $p<0.05$; Fig. 3 A,B).

During early pregnancy, the lowest expression of visfatin gene was observed on days 27 28 , however between days 10-11, 12-13 and 15-16 it was gradually increasing. Concerning the protein of visfatin, the highest concentration was observed on days 10-11 in relation to days 15-16 ( $p<0.05$; Fig. 3 C,D).

Comparing visfatin gene expression throughout the early pregnancy with days 10-12 of the estrous cycle, visfatin mRNA content during all periods of pregnancy was significantly lower. However, visfatin protein content in pregnant MBH samples was at similar level as on days $10-12$ of the cycle ( $p<0.05$; Fig. 3 E,F).

\subsection{Gene and protein expression of visfatin in the preoptic area}

During the estrous cycle, visfatin gene expression was the highest on days 17-19, lower on days 14-16, and the lowest on days 2-3 and 10-12. Concerning the protein level of visfatin, the highest content was noted on days 14-16, lower on days 2-3, and the lowest on days 10-12 and 17-19 ( $p<0.05$; Fig. 4 A,B). 
During early pregnancy, the highest expression of visfatin gene was observed on days 1516 , and the lowest on days 12-13. In turn, the protein concentrations were the highest on days 27-28, and the lowest on days 10-11 and 15-16 of gestation ( $p<0.05$; Fig. 4 C,D)

Comparing the studied periods of early pregnancy with days 10-12 of the estrous cycle, we noted that visfatin gene expression on days 15-16, 27-28 and 10-11 of pregnancy was significantly enhanced, while the expression on days 12-13 of pregnancy was considerably lower compared to this phase of the estrous cycle. In turn, visfatin protein content on days $27-$ 28 of pregnancy was essentially higher compared to the cycle, while during the other periods of pregnancy it was at a similar level as on days 10-12 of the cycle $(p<0.05$; Fig. 4 E,F).

\subsection{Visfatin concentrations in the blood plasma}

During the estrous cycle, significantly higher concentration of visfatin was noted on days 2-3 in relation to other studied phases of the cycle ( $p<0.05$; Fig. 5A). During early pregnancy, the highest visfatin contents in the blood plasma were observed on days 12-13 as compared to days 15 to 28 ( $p<0.05$; Fig. 5B), whereas the lowest on days 27-28 compared to days 10 to 13 . The comparison of the studied stages of early pregnancy and days 10-12 of the estrous cycle has shown that the concentrations of visfatin in the blood plasma on days 15-16 and 27-28

\section{Discussion}

The presented research was the first experiment to report the expression of visfatin gene and protein in the structures of porcine hypothalamus responsible for GnRH production during the estrous cycle and early pregnancy. Based on our immunohistochemical analyses, visfatin was localized in the nucleus and cytoplasm of cells creating both studied hypothalamic structures. These structures, including medial preoptic area, diagonal band of Broca, lateral hypothalamic area, paraventricular nucleus, periventricular zone, suprachiasmatic nucleus and mediobasal hypothalamus, are also known as the place of GnRH neurons location in the pig brain [31,32]. The expression pattern of visfatin gene in $\mathrm{MBH}$ and POA during the cycle and pregnancy was not parallel with the pattern of visfatin protein expression. Taking into account that in mammals the correlation between gene and protein expression frequently does not exceed $40 \%$ [33], this observation is not surprising. The mentioned phenomenon can be attributed to transcriptional, post-transcriptional and translational regulations and, as a consequence, discrepancies in mRNAs and proteins stability $[34,35]$. 
In previous studies, visfatin gene expression was noticed in the mouse and chicken hypothalamus [4, 36], analyzed however as a whole, without division into particular hypothalamic structures. Visfatin expression was also found in the pituitary of male sheep [5] and female mice [4]. In the mouse pituitary, visfatin was present mainly in gonadotroph cells, responsible for the follicle-stimulating hormone (FSH) and luteinizing hormone (LH) production [4]. To our best knowledge, this is the first study investigating visfatin gene and protein expression in the hypothalamus coupled with consideration of physiological status/hormonal milieu of animals characteristic for the estrous cycle and early pregnancy. The crucial hormonal factors related to the estrous cycle are progesterone $\left(\mathrm{P}_{4}\right)$ and estradiol $\left(\mathrm{E}_{2}\right)$. Most of them are ovarian origin, but minority is produced in the brain, including hypothalamus, as neurosteroids [37]. Thus the local concentration of steroids in the hypothalamus is a mixture of peripherally derived steroid hormones, converted peripheral steroids and neurosteroids [38, 39]. Both neurons, oligodendrocytes and astrocytes are steroidogenically active cells and their primary steroidogenic product is neuroprogesterone [37]. It was indicated that astrocytes are the main source of the essential neuroprogesterone generated within the hypothalamic structures [40]. Expression pattern of visfatin gene and protein expression in $\mathrm{MBH}$ and POA is different throughout the estrous cycle and early pregnancy, which may suggest that the regulation of visfatin generation in both hypothalamic structures is also different. It seems possible that the observed in the $\mathrm{MBH}$ an increase of visfatin protein content on days 14-16 and 17-19 of the cycle is caused by stimulatory action of $E_{2}$, which plasma level is enhanced. In turn in the POA, the noticed suppressed visfatin expression on days 10-12 and 17-19 of the cycle can be coupled with $\mathrm{P}_{4}$ concentrations in the blood and hypothalamus. On days 10-12 of the porcine estrous cycle, plasma level of $\mathrm{P}_{4}$ is the highest which may suggest that hypothalamic concentration of this steroid is also enhanced, whereas on days 17-19 the same inhibitory effect is achieved by $\mathrm{P}_{4}$ produced mainly in the hypothalamus. Studies by Micevych et al. [41] indicated that during proestrus the increased level of estrogens significantly induces neuroprogesterone synthesis in the hypothalamus. Circulating $\mathrm{E}_{2}$ stimulated also hypothalamic mRNA level and activity of $3 \beta$-hydroxysteroid

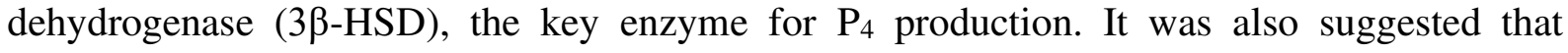
locally synthesized hypothalamic neuroprogesterone, apart from its action via progesterone receptors (PR), may be $5 \alpha$-reduced, converted to allopregnanolone, and bind to $\mathrm{GABA}_{\mathrm{A}}$ receptors $\left(\mathrm{GABA}_{\mathrm{A}} \mathrm{R}\right)$ [41]. It is postulated that this kind of $\mathrm{GABA}_{\mathrm{A}} \mathrm{R}$ stimulation is involved in the induction of GnRH release [42].

It is strongly suggested that regulatory mechanism controlling visfatin expression is tissue- and species-specific $[9,36]$. The relationship between visfatin expression in particular 
tissues and hormonal milieu of the organism is to a high degree unclear. On one hand, visfatin concentration in cerebrospinal fluid did not differ between man and women [43]. Similarly, there was no significant differences in visfatin mRNA expression in the mouse pituitary between the estrous and diestrus phases [4]. Generally, these reports indicate lack of any dependence of visfatin generation on hormonal status of the body, especially on the influence of sex steroid hormones. On the other hand, considerable evidence has accumulated to implicate the involvement of hormonal environment in the control of visfatin expression. It has been indicated that human maternal plasma concentration of visfatin was increased during pregnancy, especially between the two last and the first trimester [14]. One of reasons of the observed increase may be many fold higher visfatin gene expression in omental fat of pregnant women at term compared to nonpregnant subjects [13]. Similarly in rats, visfatin mRNA content was elevated in the white fat on day 21 of pregnancy [44]. The results of our study also indicate the relationship of pregnancy with plasma visfatin level. Our observations are slightly different compared to the mentioned above studies - we noticed a decrease in plasma visfatin concentrations on days of implantation in relation to earlier days of pregnancy and days 10-12 of the estrous cycle. The reason of these differences may be, of course, varied and species-dependent regulation of visfatin production throughout gestation, but the simplest explanation of the observed inconsistencies is distinct (very early in the case of our studies) period of pregnancy taken into consideration in our and cited above works. Additional examples were obtained based on experiments with 3T3-L1 pre-adipocytes and adipocytes. Studies with pre-adipocytes have shown that visfatin gene expression decreased under influence of insulin, $\mathrm{P}_{4}$ and testosterone and increased in response to dexamethasone [12]. In turn, treatment of 3T3-L1 adipocytes with $\mathrm{GH}, \mathrm{TNF} \alpha$, cyclic adenosine monophosphate (cAMP) and beta-adrenergic agonists caused downregulation of the adipokine expression [11], whereas the effect of sex hormones was insignificant [12]. More detailed studies concerning possible influence of sex steroid hormones on visfatin expression in adipocytes indicated that estriol added to cell cultures increased visfatin mRNA concentration and the effect of $\mathrm{P}_{4}$ and $\mathrm{E}_{2}$ was negligible, but these three steroid used in combination essentially enhanced the gene expression [45]. Moreover, unlike the mouse pituitary, visfatin expression in the mouse uterus fluctuated during the estrous cycle. It has been shown that visfatin protein concentration was increased in proestrus and metestrus, and decreased in estrus and diestrus. According to the authors, the reason of the observed changes can be the increased plasma estrogens levels in proestrus and their decrease in diestrus. Simultaneously, treatment with $\mathrm{E}_{2}$ of ovariectomized mice caused visfatin up-regulation, whereas $\mathrm{P}_{4}$ down-regulated visfatin expression. The co-treatment with both steroids enhanced visfatin protein content in the 
ovariectomized mouse uterus [46]. Taken together, the sum of our results and the results reported above suggest that expression of the visfatin gene may be hormonally regulated.

It has been suggested that besides local synthesis, additional source of visfatin in the central nervous system may be transport across the blood-brain barrier. However, the concentrations of visfatin in human cerebrospinal fluid are at the level of approximately $10 \%$ of those in plasma. Moreover, visfatin levels in plasma and cerebrospinal fluid were negatively correlated as visfatin concentrations in cerebrospinal fluid decreased with increasing plasma visfatin contents in obese subjects [43]. Similarly in our study, the concentration pattern of visfatin in both examined hypothalamic structures and in the plasma of pigs is completely different. It may suggest that the transport across the blood-brain barrier transport does not play a major role, and the local hypothalamic regulation of visfatin production is autonomic and distinct in relation to other tissues, especially the adipose tissue which seems to be the main source of the adipokine.

The visfatin role in the hypothalamus is to a high degree unknown. As previously mentioned, visfatin is both an intracellular enzyme and a secreted hormone. Especially its localization in cell nuclei seems to be intriguing and may suggest, based on data provided by Svoboda et al. [47], that NAMPT, through stimulation of NAD biosynthesis and sirtuins activation, takes part in the regulation of DNA repair, chromatin structure, transcription, replication, telomerase length and circadian rhythm. It has been indicated day-night differences of visfatin expression in the ovine pars tuberalis and an increased visfatin expression, under melatonin influence, in this structure connecting the hypothalamus and pituitary [5]. It seems that links between energy sensing and NAD cycle, and the regulation of circadian clock function exist also in other parts of the central nervous system [48]. Visfatin, acting at the hypothalamic level as an energy metabolism sensor, may be involved in the control of food intake, principally fasting- and ghrelin-induced food intake [49]. Other studies, using visfatin knockin mice, indicated an enhanced $\mathrm{NAD}^{+}$levels in multiple tissues, including the hypothalamus, and an improvement in physical activity, sleep quality and cognitive functions [50] Nevertheless, visfatin effect on pituitary secretory functions remains unknown. Its expression in the structures responsible for GnRH production may suggest that visfatin is also engaged in the control of GnRH generation. This hypothesis, however, should be confirmed in further detailed studies

\section{Conclusion}

Summarizing, visfatin expression in the porcine hypothalamic structures responsible for $\mathrm{GnRH}$ production implies its autocrine/paracrine influence on $\mathrm{GnRH}$ synthesis and confirms 
453 the potential role of visfatin as neuromodulator of reproductive functions. The variations in 454 the expression levels of visfatin noticed during the estrous cycle and early pregnancy may 455 suggest the effect of steroid hormones, both peripheral and hypothalamic origin. It cannot be 456 ruled out either, that the influence of steroids is achieved through their action on hypothalamic 457 neural systems, like GABAergic, dopaminergic, serotoninergic systems engaged in the 458 control of GnRH production. Further research focused on steroid hormones and their 459 receptors concentrations in particular hypothalamic nuclei involved in GnRH synthesis are 460 also required.

461

462

463

464

465

466

467

468

469

470

471

472

473

474

475

476

477

478

479

480

481

482

483

484

485

486 
490 18sRNA - 18S ribosomal RNA

$4913 \beta$-HSD - 3 $\beta$-hydroxysteroid dehydrogenase

492 ARC - arcuate nucleus

493 BSA - bovine serum albumin

494 cAMP - cyclic adenosine monophosphate

495 DBB - diagonal band of Broca

$496 \quad E_{2}$ - estradiol

497 ELISA - enzyme-linked immunosorbent assay

498 FSH - follicle-stimulating hormone

$499 \mathrm{GABA}_{\mathrm{A}} \mathrm{R}-\mathrm{GABA}_{\mathrm{A}}$ receptors

$500 \mathrm{GH}$ - growth hormone

501 GnRH - gonadotropin releasing hormone

502 hCG - human chorionic gonadotropin

503 HPG - hypothalamic-pituitary-gonadal axis

504 INSR - insulin receptor

505 LH - luteinizing hormone

506 LPA - lateral preoptic area

$507 \mathrm{MBH}$ - mediobasal hypothalamus

508 MPA - medial preoptic area

509 NAD - nicotinamide adenine dinucleotide

510 NAMPT - nicotinamide phosphoribosyltransferase

$511 \mathrm{NaN}_{3}$ - sodium azide

$512 \mathrm{P}_{4}$ - progesterone

513 PBS - phosphate-buffered saline

514 POA - preoptic area of the hypothalamus

515 PPN - periventricular nucleus

516 PR - progesterone receptors

517 PVN - paraventricular nucleus

518 qPCR - quantitative real-time PCR

$519 \mathrm{SCN}$ - suprachiasmatic nucleus

520 SDN - sexually dimorphic nucleus of the preoptic area

521 SEM - standard error of the mean

522 SON - supraoptic nucleus 
523 TBST - Tris-buffered saline with $0.1 \%$ Tween ${ }^{\circledR} 20$ Detergent

$524 \mathrm{TNF} \alpha-$ tumor necrosis factor $\alpha$

525 UBC - ubiquitin C

526 VMH - ventromedial nucleus

527

528

529

530

531

532

533

534

535

536

537

538

539

540

541

542

543

544

545

546

547

548

549

550

551

552

553

554

555

556

557 


\section{Bibliography}

559

560

1. Fukuhara A, Matsuda M, Nishizawa M, Segawa K, Tanaka M, Kishimoto K, et al. Visfatin:

561 a protein secreted by visceral fat that mimics the effects of insulin. Science. 2005;307:426-31.

562 2. Xie H, Tang SY, Luo XH, Huang J, Cui RR, Yuan LQ, et al. Insulin-like effects of visfatin

563 on human osteoblasts. Calcif Tissue Int. 2007;80:201-10.

564 3. Adeghate E. Visfatin: Structure, Function and Relation to Diabetes Mellitus and Other 565 Dysfunctions. Curr Med Chem. 2008;15:1851-62.

566

4. Maillard V, Elis S, Desmarchais A, Hivelin C, Lardic L, Lomet D, et al. Visfatin and 567 resistin in gonadotroph cells: Expression, regulation of LH secretion and signalling pathways.

568 Reprod Fertil Dev. 2017;29:2479-95.

569

5. Dupré SM, Burt DW, Talbot R, Downing A, Mouzaki D, Waddington D, et al. 570 Identification of melatonin-regulated genes in the ovine pituitary pars tuberalis, a target site for seasonal hormone control. Endocrinology. 2008;149:5527-39.

572 6. Shen CJ, Tsai EM, Lee JN, Chen YL, Lee CH, Chan TF. The concentrations of visfatin in 573 the follicular fluids of women undergoing controlled ovarian stimulation are correlated to the number of oocytes retrieved. Fertil Steril. 2010;93:1844-50. 7. Reverchon M, Cornuau M, Cloix L, Ramé C, Guerif F, Royère D, Dupont J. Visfatin Is

576 Expressed in Human Granulosa Cells: Regulation by Metformin Through AMPK/SIRT1 577 Pathways and Its Role in Steroidogenesis. Mol Hum Reprod. 2013;19.

578 8. Reverchon M, Rame C, Bunel A, Chen W, Froment P, Dupont J. Visfatin (NAMPT) 579 Improves in Vitro IGF1-Induced Steroidogenesis and IGF1 Receptor Signaling Through 580 SIRT1 in Bovine Granulosa Cells1. Biol Reprod. 2016;94.

581 9. Palin MF, Labrecque B, Beaudry D, Mayhue M, Bordignon V, Murphy BD. Visfatin 582 expression is not associated with adipose tissue abundance in the porcine model. Domest 583 Anim Endocrinol. 2008;35:58-73.

584 10. Barbe A, Kurowska P, Mlyczyńska E, Ramé C, Staub C, Venturi E, et al. Adipokines 585 expression profiles in both plasma and peri renal adipose tissue in Large White and Meishan 586 sows: a possible involvement in the fattening and the onset of puberty. Gen Comp Endocrinol. 587 2020;299:113584.

588 11. Kralisch S. Hormonal regulation of the novel adipocytokine visfatin in 3T3-L1 589 adipocytes. J Endocrinol. 2005;185:R1-8. 
12. MacLaren R, Cui W, Cianflone K. Visfatin expression is hormonally regulated by metabolic and sex hormones in 3T3-L1 pre-adipocytes and adipocytes. Diabetes Obes Metab. 2007;9:490-7.

13. Morgan SA, Bringolf JB, Seidel ER. Visfatin expression is elevated in normal human pregnancy. Peptides. 2008;29:1382-9.

14. Mastorakos G, Valsamakis G, Papatheodorou DC, Barlas I, Margeli A, Boutsiadis A, et al. The role of adipocytokines in insulin resistance in normal pregnancy: visfatin concentrations in early pregnancy predict insulin sensitivity. Clin Chem. 2007;53:1477-83. 15. Berndt J, Klöting N, Kralisch S, Kovacs P, Fasshauer M, Schön MR, et al. Plasma visfatin concentrations and fat depot-specific mRNA expression in humans. Diabetes. 2005;54:29116.

16. Filippatos TD, Derdemezis CS, Kiortsis DN, Tselepis AD, Elisaf MS. Increased plasma levels of visfatin/pre-B cell colony-enhancing factor in obese and overweight patients with metabolic syndrome. J Endocrinol Invest. 2007;30:323-6.

17. Reverchon M, Ramé C, Bertoldo M, Dupont J. Adipokines and the female reproductive tract. Int J Endocrinol. 2014; 232454.

18. Hug C. Visfatin: a new adipokine. Science. 2005;307:366-7.

19. Kiezun M, Smolinska N, Maleszka A, Dobrzyn K, Szeszko K, Kaminski T. Adiponectin expression in the porcine pituitary during the estrous cycle and its effect on $\mathrm{lh}$ and fsh secretion. Am J Physiol - Endocrinol Metab. 2014;307:E1038-46.

20. Cloix L, Reverchon M, Cornuau M, Froment P, Ramé C, Costa C, et al. Expression and regulation of INTELECTIN1 in human granulosa-lutein cells: role in IGF-1-Induced steroidogenesis through NAMPT1. Biol Reprod. 2014;91.

21. Dupont J, Pollet-Villard X, Reverchon M, Mellouk N, Levy R. Adipokines in human reproduction. Horm Mol Biol Clin Investig. 2015;24:11-24.

22. Różycka M, Kurowska P, Grzesiak M, Kotula-Balak M, Tworzydło W, Rame C, et al. Apelin and apelin receptor at different stages of corpus luteum development and effect of apelin on progesterone secretion and $3 \beta$-hydroxysteroid dehydrogenase (3 $\beta$-HSD) in pigs. Anim Reprod Sci. 2018;192:251-60.

23. Choi KH, Joo BS, Sun ST, Park MJ, Son J Bin, Joo JK, et al. Administration of visfatin during superovulation improves developmental competency of oocytes and fertility potential in aged female mice. Fertil Steril. 2012;97.

24. Mazaki-Tovi S, Romero R, Vaisbuch E, Kim SK, Kusanovic JP, Chaiworapongsa T, et al. Evidence for differential regulation of the adipokine visfatin in the maternal and fetal compartments in normal spontaneous labor at term. J Perinat Med. 2010;38:281-8. 
25. Akins EL, Morrissette MC. Gross ovarian changes during estrous cycle of swine. Am J Vet Res. 1968;29:1953-7.

26. Anderson LL. Growth, protein content and distribution of early pig embryos. Anat Rec. 1978;190:143-53.

27. Sesti LAC, Britt JH. Relationship of secretion of GnRH in vitro to changes in pituitary concentrations of LH and FSH and serum concentrations of LH during lactation in sows. $\mathrm{J}$ Reprod Fertil. 1993;98:393-400.

28. Félix B, Léger ME, Albe-Fessard D, Marcilloux JC, Rampin O, Laplace JP, et al. Stereotaxic atlas of the pig brain. Brain Res Bull. 1999;49:1-137.

29. Smolinska N, Dobrzyn K, Kiezun M, Szeszko K, Maleszka A, Kaminski T. En, progesterone and androstenedione production by the porcine uterus during early pregnancyffect of adiponectin on the steroidogenic acute regulatory protein, p450 side chain cleavage enzyme and 3B-hydroxysteroid dehydrogenase gene expressio. J Physiol Pharmacol. 2016;67:443-56.

30. Livak KJ, Schmittgen TD. Analysis of relative gene expression data using real-time quantitative PCR and the 2- $\Delta \Delta C$ T method. Methods. 2001;25:402-8.

31. Kineman RD, Leshin LS, Crim JW, Rampacek GB, Kraeling RR. Localization of luteinizing hormone-releasing hormone in the forebrain of the pig. Biol Reprod. 1988;39:665-72.

32. Polkowska J, Liwska J, Dubois MP. Ontogeny of neuropeptidergic systems: Luteinizing hormone releasing hormone (LHRH): Somatostatin (SRIF) and neurophysin (NF) in the hypothalamus of the domestic pig by immunocytochemistry. Folia Histochem Cytobiol. 1985;23:193-200.

33. De Sousa Abreu R, Penalva LO, Marcotte EM, Vogel C. Global signatures of protein and mRNA expression levels. Molecular BioSystems. 2009;5:1512-26.

34. Gry M, Rimini R, Strömberg S, Asplund A, Pontén F, Uhlén M, et al. Correlations between RNA and protein expression profiles in 23 human cell lines. BMC Genomics. 2009; 10:365.

35. Vogel C, Marcotte EM. Insights into the regulation of protein abundance from proteomic and transcriptomic analyses. Nat Rev Genet. 2012;13:227-32.

36. Ons E, Gertler A, Buyse J, Lebihan-Duval E, Bordas A, Goddeeris B, et al. Visfatin gene expression in chickens is sex and tissue dependent. Domest Anim Endocrinol. 2010;38:63-74. 37. Schumacher M, Hussain R, Gago N, Oudinet JP, Mattern C, Ghoumari AM. Progesterone synthesis in the nervous system: Implications for myelination and myelin repair. Frontiers in Neuroscience. 2012; 6:10. 
38. Micevych P, Sinchak K. Minireview: Synthesis and function of hypothalamic neuroprogesterone in reproduction. Endocrinology. 2008;149:2739-42.

39. Rossetti MF, Cambiasso MJ, Holschbach MA, Cabrera R. Oestrogens and Progestagens: Synthesis and Action in the Brain. J Neuroendocrinol. 2016;28.

40. Kuo J, Micevych P. Neurosteroids, trigger of the LH surge. J Steroid Biochem Mol Biol. 2012;131:57-65.

41. Micevych P, Sinchak K, Mills RH, Tao L, LaPolt P, Lu JKH. The luteinizing hormone surge is preceded by an estrogen-induced increase of hypothalamic progesterone in ovariectomized and adrenalectomized rats. Neuroendocrinology. 2003;78:29-35.

41. Hallschmid M, Randeva H, Tan BK, Kern W, Lehnert H. Relationship between cerebrospinal fluid visfatin (PBEF/Nampt) levels and adiposity in humans. Diabetes. 2009;58:637-40.

42. Graham JD, Clarke CL. Physiological Action of Progesterone in Target Tissues. Endocr Rev. 1997;18:502-19.

43. Josephs T, Waugh H, Kokay I, Grattan D, Thompson M. Fasting-induced adipose factor identified as a key adipokine that is up-regulated in white adipose tissue during pregnancy and lactation in the rat. J Endocrinol. 2007;194:305-12.

44. Zhou J, Seidel ER. Estrogens induce visfatin expression in 3T3-L1 cells. Peptides. 2010;31:271-4.

45. Annie L, Gurusubramanian G, Roy VK. Estrogen and progesterone dependent expression of visfatin/NAMPT regulates proliferation and apoptosis in mice uterus during estrous cycle. J Steroid Biochem Mol Biol. 2019;185:225-36.

46. Svoboda P, Krizova E, Sestakova S, Vapenkova K, Knejzlik Z, Rimpelova S, et al. Nuclear transport of nicotinamide phosphoribosyltransferase is cell cycle- dependent in mammalian cells, and its inhibition slows cell growth. J Biol Chem. 2019;294:8676-89.

47. Reick M, Garcia JA, Dudley C, McKnight SL. NPAS2: An analog of clock operative in the mammalian forebrain. Science. 2001;293:506-9.

48. de Guia RM, Hassing AS, Skov LJ, Ratner C, Plucińska K, Madsen S, et al. Fasting- and ghrelin-induced food intake is regulated by NAMPT in the hypothalamus. Acta Physiol. 2020;228.

49. Yoshida M, Satoh A, Lin JB, Mills KF, Sasaki Y, Rensing N, et al. Extracellular vesiclecontained eNAMPT delays aging and extends lifespan in mice. Cell Metab. 2019;30:329342.e5. 
693 50. Szeszko K, Smolinska N, Kiezun M, Dobrzyn K, Maleszka A, Kaminski T. The influence 694 of adiponectin on the transcriptomic profile of porcine luteal cells. Funct Integr Genomics. 695 2016;16:101-14.

696 51. Smolinska N, Kiezun M, Dobrzyn K, Rytelewska E, Kisielewska K, Gudelska M, et al. 697 Expression of chemerin and its receptors in the porcine hypothalamus and plasma chemerin 698 levels during the oestrous cycle and early pregnancy. Int J Mol Sci. 2019;20.

699

700

701

702

703

704

705

706

707

708

709

710

711

712

713

714

715

716

717

718

719

720

721

722

723

724

725

726 


\section{Figures}
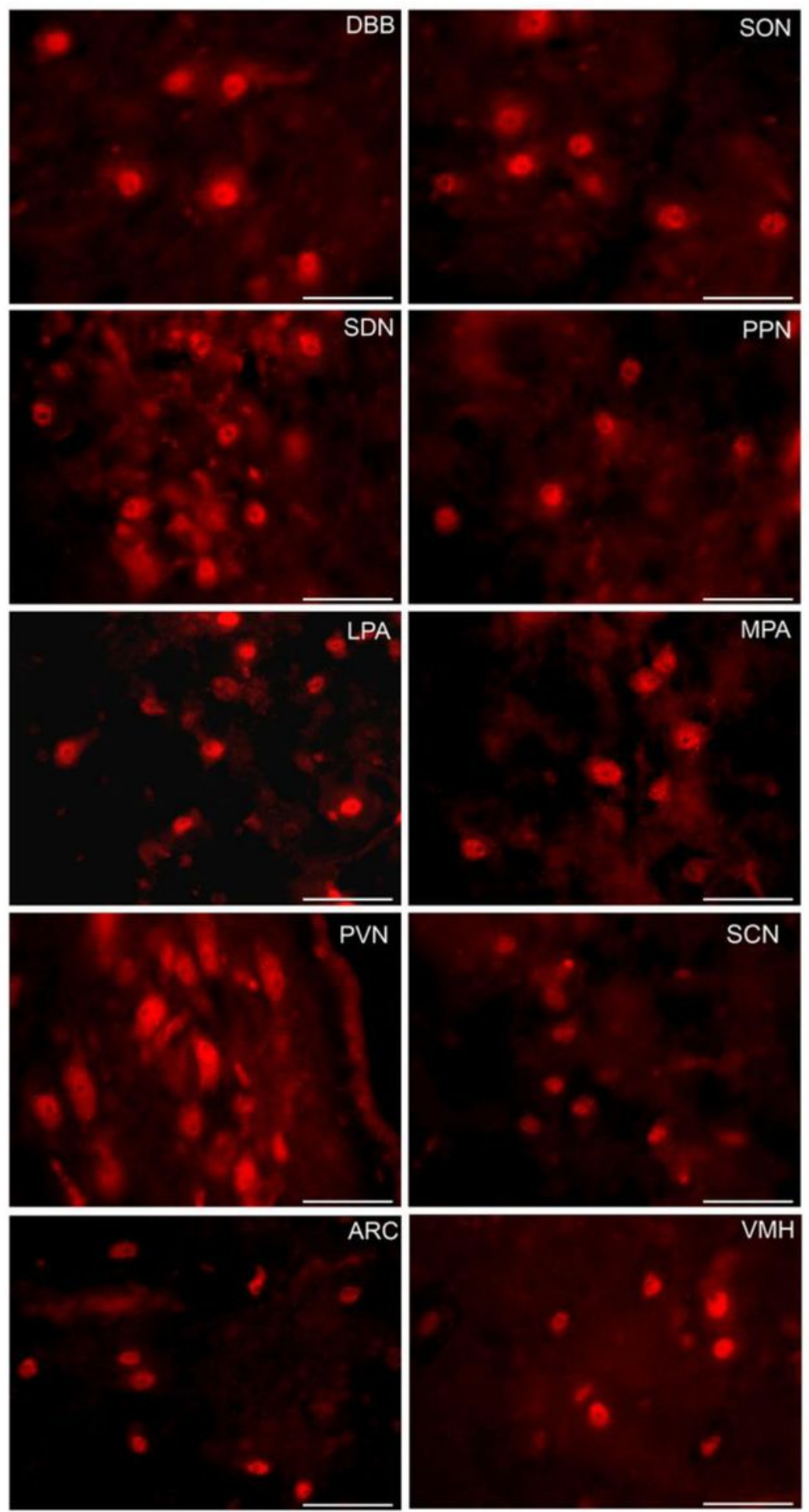

$\mathrm{VMH}$

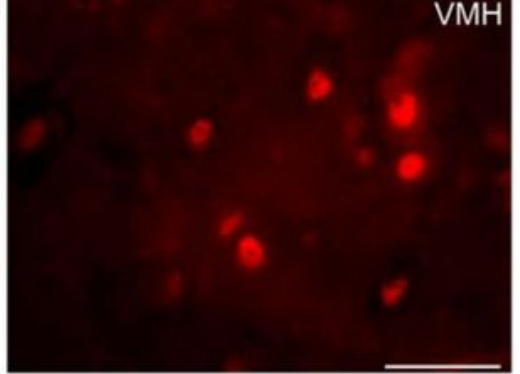

\section{Figure 1}

The visfatin localization in the porcine hypothalamus on days 10-12 of the estrous cycle Immunoreactivity of visfatin was determined by fluorescent immunohistochemistry. Particular images indicate the immunolocalization of visfatin in the diagonal band of Broca (DBB), supraoptic nucleus 
(SON), preoptic area (SDN), periventricular nucleus (PPN), lateral (LPA) and medial preoptic area (MPA), paraventricular nucleus (PVN), suprachiasmatic nucleus (SCN), arcuate nucleus (ARC), as well as ventromedial nucleus $(\mathrm{VMH})$. Scale bar: $50 \mu \mathrm{m}$.
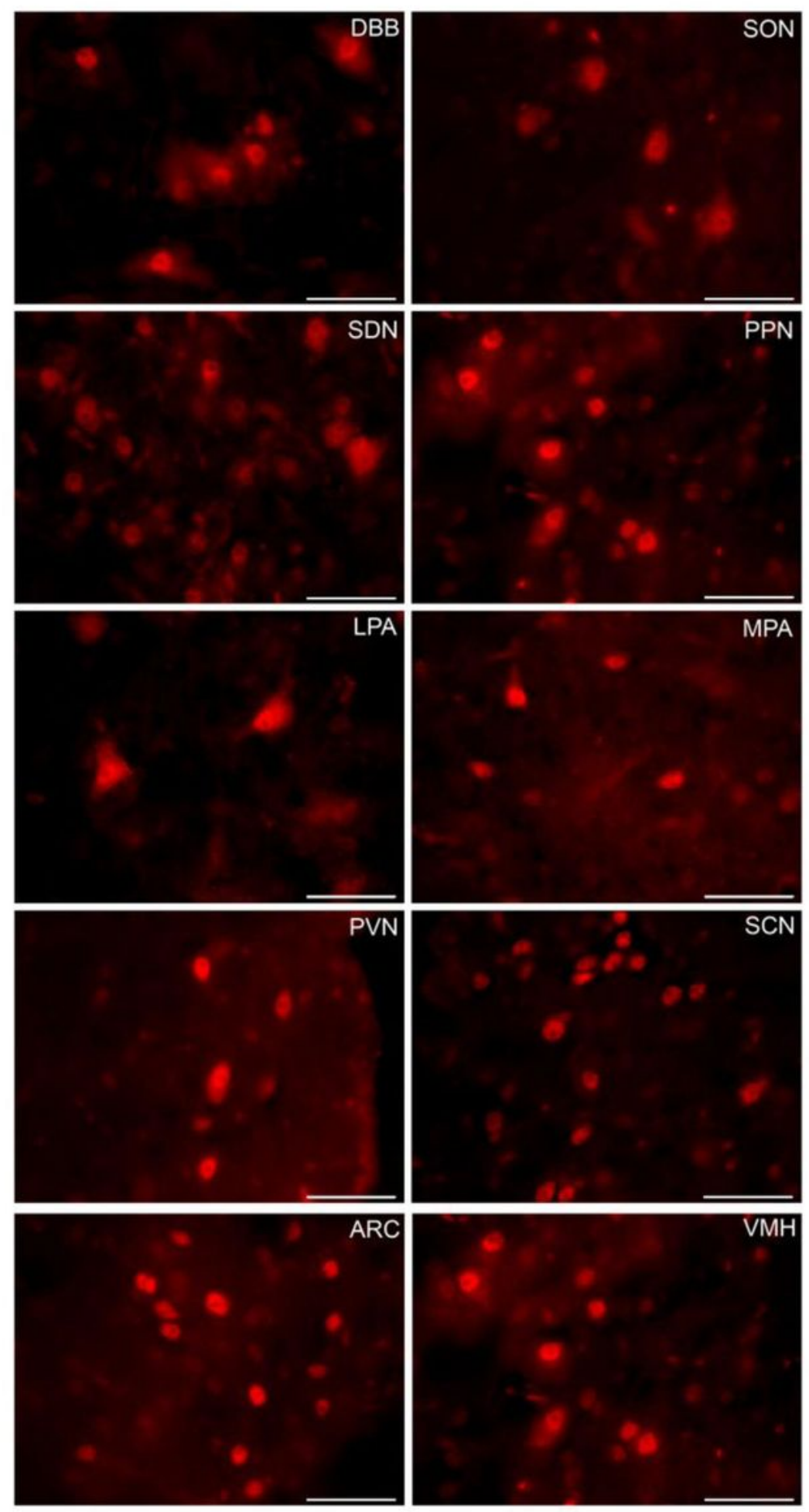

Figure 2

The visfatin localization in the porcine hypothalamus on days 15-16 of early pregnancy Immunoreactivity of visfatin was determined by fluorescent immunohistochemistry. Particular images indicate the 
immunolocalization of visfatin in the diagonal band of Broca (DBB), supraoptic nucleus (SON), preoptic area (SDN), periventricular nucleus (PPN), lateral (LPA) and medial preoptic area (MPA), paraventricular nucleus (PVN), suprachiasmatic nucleus ( $\mathrm{SCN})$, arcuate nucleus (ARC), as well as ventromedial nucleus (VMH). Scale bar: $50 \mu \mathrm{m}$.

RELATIVE GENE EXPRESSION
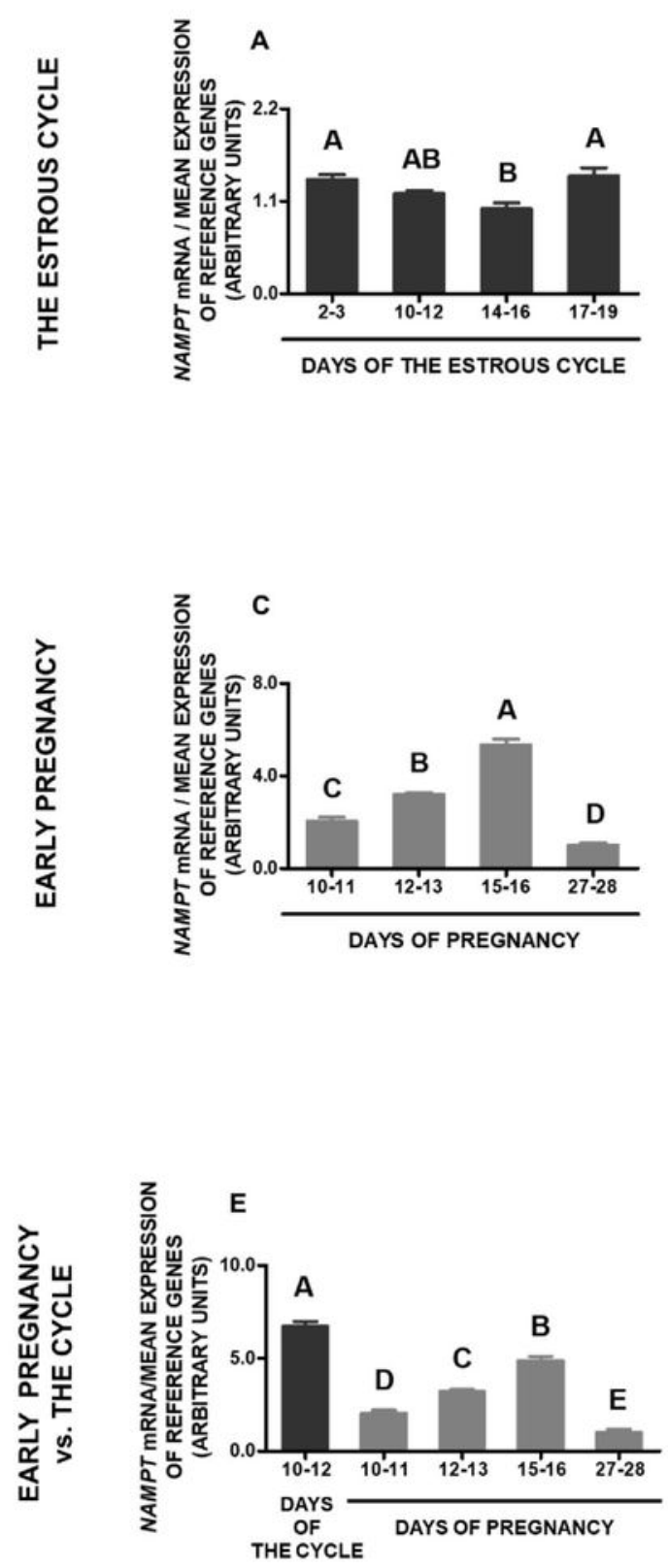

RELATIVE PROTEIN EXPRESSION
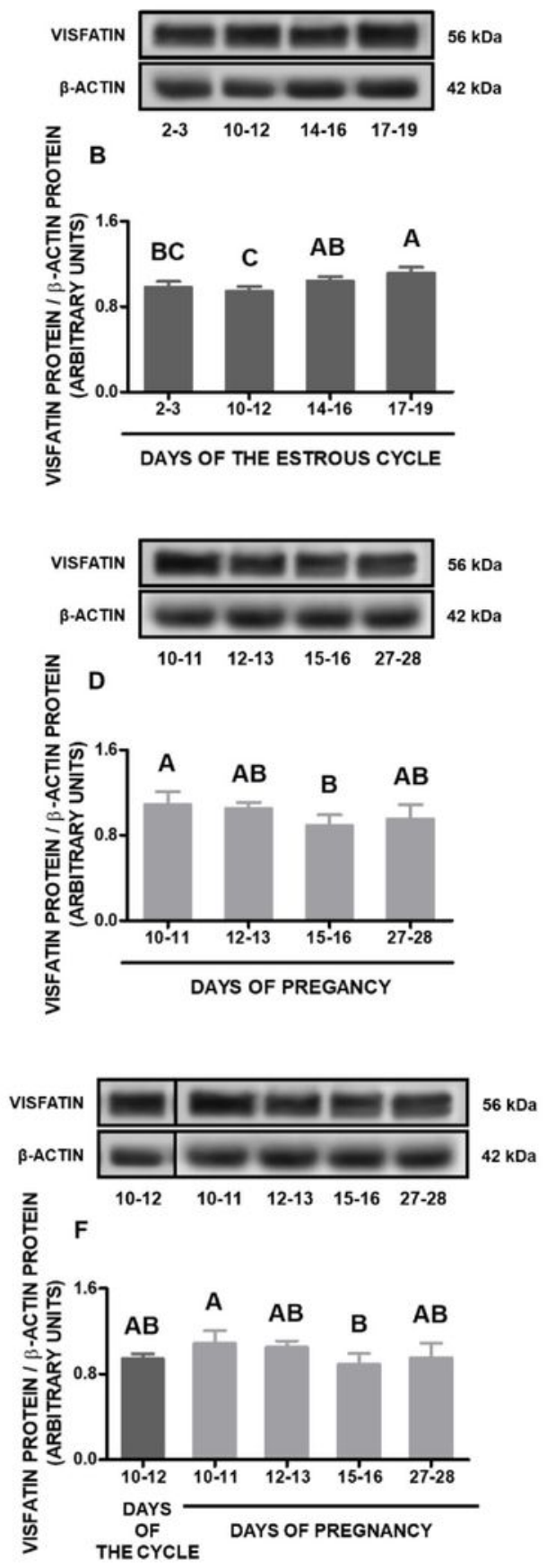

Figure 3 
Visfatin gene and protein expression in the porcine mediobasal hypothalamus $(\mathrm{MBH})$ during the estrous cycle and early pregnancy Gene and protein expression of visfatin in the porcine $\mathrm{MBH}$ was determined during the estrous cycle on days: 2-3, 10-12, 14-16 and 17-19 (A, B), during early pregnancy on days: 10$11,12-13,15-16$ and 27-28 (C, D) and compared between early pregnancy and days 10-12 of the estrous cycle $(E, F)$. Gene expression was analyzed by qPCR. Protein expression was analyzed by Western blotting; upper panels: representative immunoblots; lower panels: densitometric analyzis of visfatin protein relative to actin protein. Results are presented as means \pm SEM $(n=5)$. Bars with different superscripts are significantly different (one-way ANOVA at $p<0.05$ followed by Tuckey post hoc test at $p<0.05)$. 

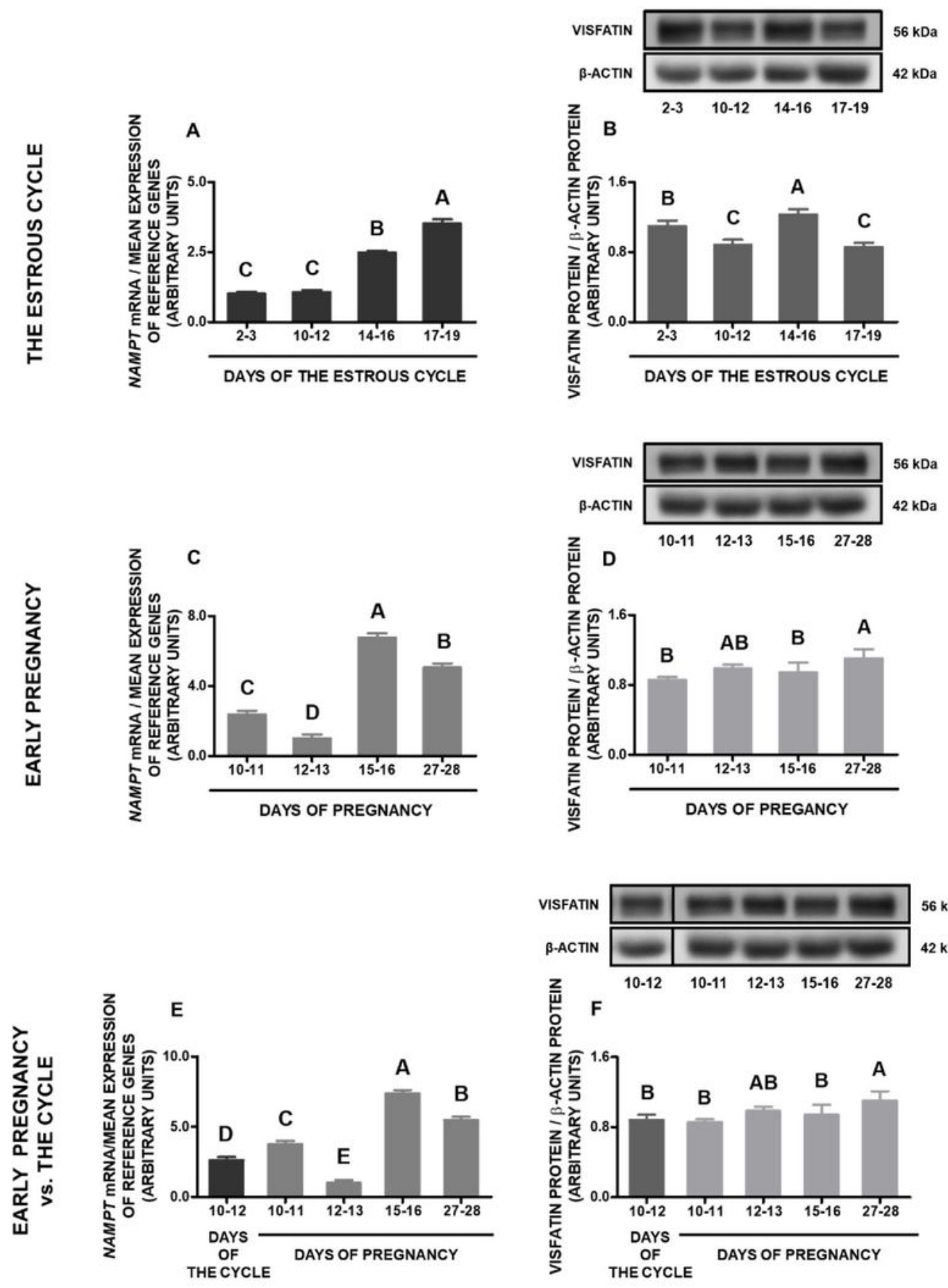

\section{Figure 4}

Visfatin gene and protein expression in the porcine preoptic area (POA) during the estrous cycle and early pregnancy Gene and protein expression of visfatin in the porcine POA was determined during the estrous cycle on days: $2-3,10-12,14-16$ and 17-19 (A, B), during early pregnancy on days: 10-11, 12-13, $15-16$ and 27-28 (C, D) and compared between early pregnancy and days 10-12 of the estrous cycle (E, F). Gene expression was analyzed by qPCR. Protein expression was analyzed by Western blotting; upper panels: 
representative immunoblots; lower panels: densitometric analyzis of visfatin protein relative to actin protein. Results are presented as means \pm SEM $(n=5)$. Bars with different superscripts are significantly different (one-way ANOVA at $p<0.05$ followed by Tuckey post hoc test at $p<0.05$ ).
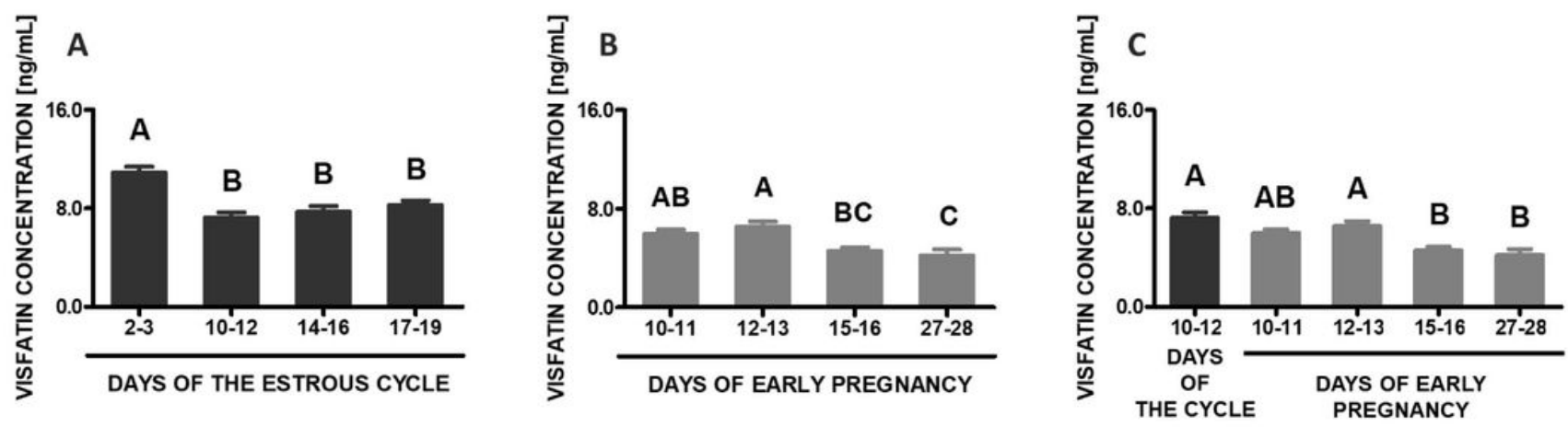

\section{Figure 5}

Visfatin concentration in the porcine blood plasma Concentrations of visfatin in the porcine blood plasma was determined during the estrous cycle on days: 2-3, 10-12, 14-16 and 17-19 (A), during early pregnancy on days: 10-11, 12-13, 15-16 and 27-28 (B) and compared between early pregnancy and days 10-12 of the estrous cycle (C). The hormone content in blood plasma was evaluated using ELISA. Results are presented as means \pm SEM $(n=5)$. Bars with different superscripts are significantly different (one-way ANOVA at $p<0.05$ followed by Tuckey post hoc test at $p<0.05$ ).

\section{Supplementary Files}

This is a list of supplementary files associated with this preprint. Click to download.

- Table1.pdf 